\title{
PERSPECTIVE
}

\section{Fundal white dots: the spectrum of a similar pathological process}

\author{
David Ben Ezra, John V Forrester
}

The intermittent appearance and subsequent vanishing of subtle white dots observed in otherwise healthy young women was described as a specific clinical entity and given the name multiple evanescent white dot syndrome (MEWDS). Although originally reported as a relatively benign phenomenon without sequelae, ${ }^{1}$ choroidal neovascularisation and affection of the visual acuity were later described. ${ }^{2}$ Because the white dots in MEWDS were clinically believed to be observed mostly at the level of the pigment epithelium, it was suggested that the pathogenesis of this condition is an underlying dysfunction of the pigment epithelium and/or pigment epithelium-photoreceptor complex. ${ }^{45}$

White dots of variable sizes and configurations are frequently observed in many uveitic entities where the choroid is involved in the inflammatory process. Moreover, on careful follow up, evanescence of these white dots is observed in most (if not all) of these cases $^{6}$ (see Fig le and $f$ ). Thus, the fact that 'multiple evanescent white dots' occur in many conditions suggests that the white dot may be an expression of a general phenomenon. The pathological sequelae and clinical outcome of white dots, however, are variable. These most probably depend, on the one hand, on the specific triggering factors and, on the other hand, on individual host response and local conditions intrinsic to the affected eye(s).

Because of the scarcity of pathological specimens and the lack of reliable laboratory tests in most of these clinical entities, our assumptions regarding the process of 'white dot' formation are mostly based on clinical observations. White dots can be observed as an early disease phenomenon in the following clinical entities: discrete multifocal choroiditis (DMC), punctate inner choroidopathy (PIC), multiple evanescent white dot syndrome (MEWDS), acute blind spot enlargement (ABSE), and the big blind spot syndrome (BBSS).

Typically, the white dots in these cases are confined to the posterior pole, are small $(25$ to $100 \mu \mathrm{m})$, discrete, and do not tend to coalesce (Fig 1a). Involvement of the neuroretina and its vessels and/or the presence of inflammatory cells within the vitreous is not a major feature of these entities. On the other hand, white dots are observed as a later phenomenon of the disease course in the following clinical entities: birdshot choroidopathy, serpiginous choroiditis, acute posterior multifocal placoid pigment epitheliopathy (APMPPE), Vogt-Koyanagi-Harada disease $(\mathrm{VKH})$, and sympathetic ophthalmia. The white dots in these latter diseases, although mostly situated in the posterior pole, can be observed throughout the entire fundus. They are generally of larger and of variable size (50-500 $\mu \mathrm{m})$, do not have well delineated borders initially, and tend to coalesce (Fig 1b). Similar manifestations are also observed in cases of infectious uveitides and especially in those associated with parasitic infections such as diffuse unilateral subacute neuroretinitis (DUSN). In these latter groups of diseases, involvement of the retina and its vessels may be a prominent feature. Furthermore, the presence of inflammatory cells within the vitreous is an early and important finding in all cases.

White dots also occur in uveoretinal inflammatory disease such as sarcoid retinal vasculitis, idiopathic central retinal vasculitis, peripheral retinal vasculitis, presumed ocular histoplasmosis, and occasionally in intermediate uveitis/pars planitis in the peripheral choroid (Fig 1 c, d). In some of these conditions the white dots are evanescent (Fig 1e, f) while in others they leave a permanent scar (seen as a window defect on fluorescein angiography). In such cases the active lesion is ill defined and indistinct while the 'healed' scar, if present, is sharp edged and pale.

\section{Clinical studies of white dots}

In order to clarify these initial impressions regarding the possible spectrum of a similar phenomenon in the formation of white dots, we prospectively followed 70 patients with ocular manifestations characteristic of the above entities for a period of 6 years (Table 1). Based on the clinical behaviour of these cases we made the following observations:

(a) The milder the accompanying inflammatory signs, the more likely were the white dots to be small $(<50 \mu \mathrm{m})$, discrete, and evanescent (that is, to disappear without leaving a scar) (Fig 1a, e, f). In contrast, large white dots $(>100 \mu \mathrm{m})$, which were less sharp edged, had a higher tendency to coalesce and were associated with more severe intraocular inflammatory reactions (Fig $1 \mathrm{~b}-\mathrm{d}$ ).

(b) Untreated, small discrete white dots tended to decrease in size and after disappearing did not reappear at the same site. A white dot with poorly delineated borders, which failed to become sharp edged within 3 to 5 days of

Table 1 Clinical manifestations of white dots

\begin{tabular}{llrrlll}
\hline $\begin{array}{l}\text { Clinical } \\
\text { diagnosis }\end{array}$ & Aetiology & $N$ & $M / F$ & $\begin{array}{l}\text { Vitreous } \\
\text { haze }\end{array}$ & $\begin{array}{l}\text { Degree of } \\
\text { evanescence }\end{array}$ & Outcome \\
\hline SO & Autoimmune & 17 & $11 / 6$ & ++ & $+/-$ & Variable \\
VKH & ?viral & 11 & $3 / 8$ & ++ & + & Fair \\
APMPPE & ?viral & 9 & $3 / 6$ & + & $+/-$ & Good \\
BSC & ?immune & 8 & $0 / 8$ & $+/-$ & $+1-$ & Poor \\
MEWDS & $?$ & 8 & $3 / 5$ & - & ++ & Good \\
PIC & $?$ & 3 & $0 / 3$ & - & + & Fair/good \\
DMC & $?$ & 12 & 2 & + & + & Fair/poor \\
DUSN & Parasite & 12 & $8 / 4$ & + & ++ & Fair/good \\
\hline
\end{tabular}

SO=Sympathetic ophthalmia. VKH = Vogt-Koyanagi-Harada disease; APMPPE = acute posterior multifocal placoid pigment epitheliopathy; BSC = birdshot choroidopathy; MEWDS = multiple evanescent white dot
syndrome; PIC=punctate inner choroidopathy; DMC=discrete multifocal syndrome; $\mathrm{PIC}=$ punctate inner choroidopathy; $\mathrm{DMC}=$ discre
choroiditis; DUSN = diffuse unilateral subacute neuroretinitis. choroiditis; DUSN = diffuse unilateral subacute neuroretinitis.
${ }^{A} \mathrm{~A}$ 'good' outcome refers to a final visual acuity between $20 / 30(6 / 9)$ and $20 / 20(6 / 60) ;$ a 'fair' outcome refers to a final visual acuity between $20 / 100$
$(6 / 36)$ to $20 / 40(6 / 12)$; a 'poor' outcome refers to a final visual acuity less than $20 / 200(6 / 60)$. 

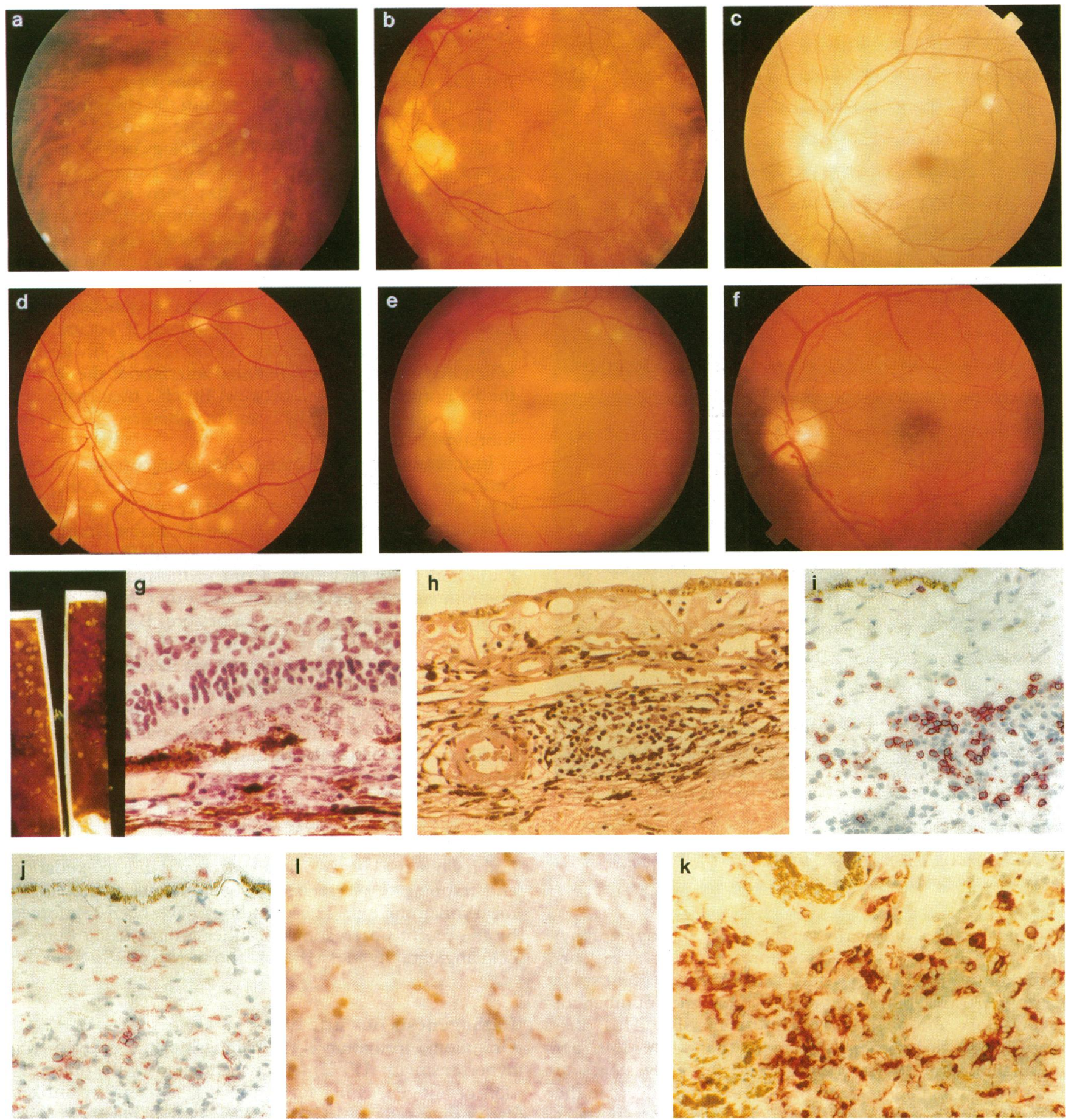

Figure 1 (a) Multiple white dots in a case of posterior uveitis; (b) multiple white dots in the deep choroid in birdshot choroidoretinopathy; (c) white dots and vitreous haze in sarcoid uveoretinitis; (d) healed white dot scars in 'presumed ocular histoplasmosis' syndrome; (e) white dots in multifocal choroiditis, (f) same case as (e) showing 'resolution' of the white dots with minimal scar formation; (g) microgranuloma in sympathetic ophthalmia at the choroidoretinal interface; inset: a macroscopic section of the globe showing numerous 'white dots' which are histologically microgranulomata; (h) deep choroidal granuloma in case of presumed sympathetic ophthalmia which clinically manifested as 'white dots'; (i) same case as (h) showing CD4+ T cells in the granuloma stained by the APAAP immunostaining technique; $(j)$ same case as $(h)$ showing low numbers of CD8+T cells in the lesion; (k) extensive positive staining for macrophages in an active granuloma in the choroid; (l) immunoperoxidase staining of a choroidal whole mount from a normal rat eye. Note the widespread distribution of the MHC class II dendritic cells in the choroid. ${ }^{28}$

onset, usually increased in size and was less prone to resolve (disappear) during the next 3 to 4 weeks.

(c) In bilateral disease, the evolving pattern and distribution of the white dots in the fundus adopted a similar course in both eyes in most cases. In a few cases small, discrete, and rapidly evanescent white dots were observed in one eye, while in the other eye the white dots tended to increase in size, coalesce, and persist. In these cases the intraocular inflammatory reaction was always more intense in the eye with the longer lasting and larger white dots.

(d) Untreated white dots had an unpredictable course in individual cases during the initial phase of the disease. If they remained discrete their 'natural course' was to resolve and disappear without treatment. However, treatment with corticosteroids and/or cyclosporin A markedly influenced the white dot configuration: they tended to decrease in size, to become more delineated, and to resolve more rapidly. This tendency correlated well with the decrease in the intensity of the intraocular inflammatory signs. However, the effect of short courses of treatment on the rate of recurrence was unpredictable.

(e) Fluorescein angiography of fundal white dots demonstrated the following: (i) patchy appearance of the 

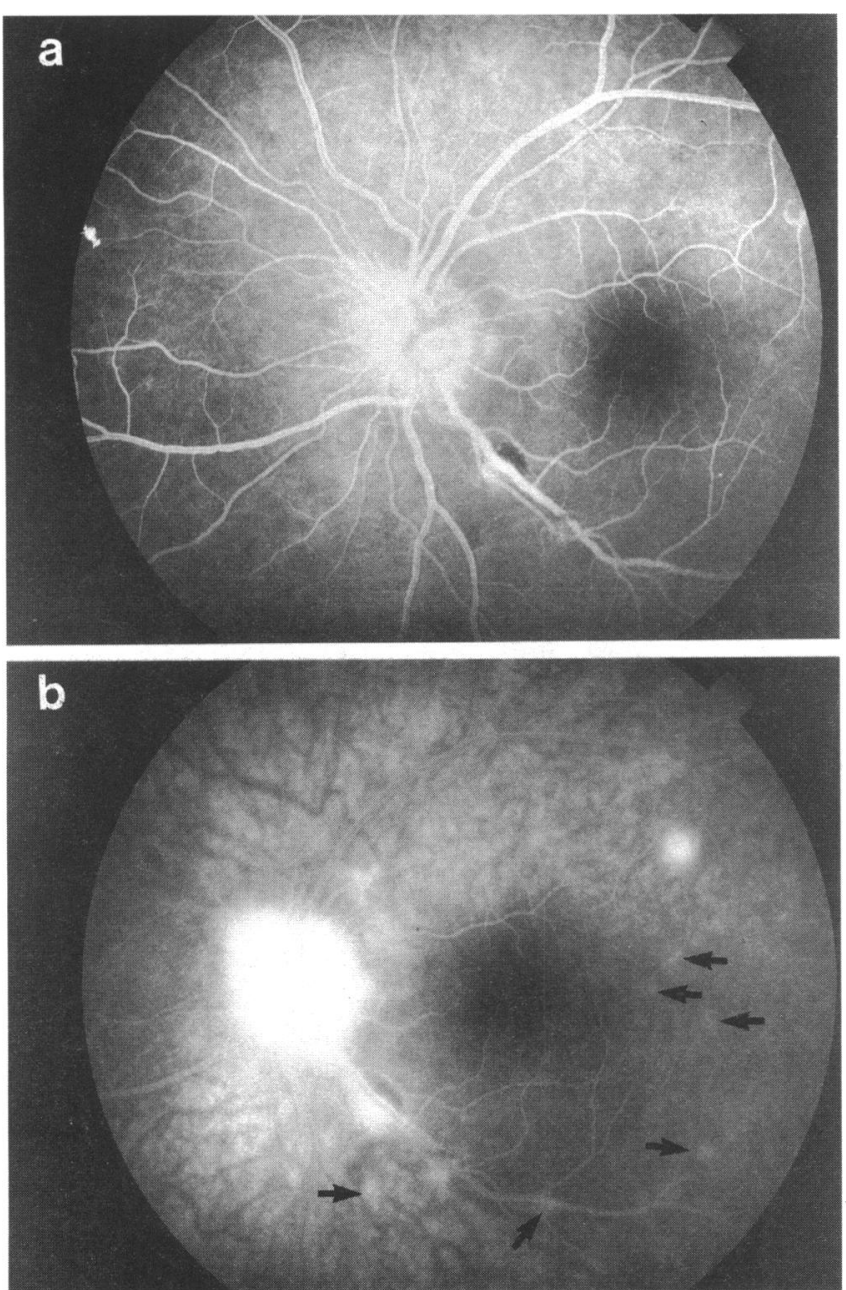

Figure 2 Fluorescein angiogram of fundus shown in patient 1(c) above. Note the patchy filling of the choroid in the early phase with areas of hypofluorescence (a) which appear as hyperfluorescent spots in the late sequence (b) (arrows). Note also that there are many more hyperfluorescent spots in the late phase than are visible as 'granulomas' on the fundal examination (see Fig 1c).

choroidal filling during the early stages of angiography; (ii) spots of choroidal hypofluorescence at sites corresponding with the white dots; (iii) hypofluorescent spots were generally more numerous than the number of white dots observed ophthalmoscopically. This discrepancy is most probably because the 'white dots' are ophthalmoscopically invisible during the early stages of their formation and/or when they are situated 'deep' in the choroid; (iv) hyperfluorescence of some spots in later stages of the angiogram (Fig 2).

In our view, these clinical and fluorescein angiography findings were indicative of a patchy inflammatory disease and were not compatible with a primary retinal pigment epithelial disorder as previously suggested ${ }^{47}$ although the retinal pigment epithelium (RPE) was frequently involved.

\section{Histology of 'white dots'}

As indicated above, white dots, both evanescent and persistent, occur in a wide range of clinically recognised syndromes. Emphasis has been placed on the ophthalmoscopic differentiation of these syndromes ${ }^{1}$ but there is considerable overlap in the clinical entities. In particular, there is commonly some degree of inflammation in the form of retinal vessel inflammation and vitreal cells. ${ }^{89}$ The main points of differentiation appear to be related to the size and locality of the white dots, both in their relation to the fovea and to the depth of the lesion within the choroid. However, as we have indicated above, the size and the persistence (or evanescence) of the lesion appear to correlate with the degree of inflammation. It would thus appear that most, if not all, white dots are manifestations of variably sized inflammatory foci at some level within the choroid. ${ }^{6} 10$

Histological studies of white dots are rare particularly in the acute stage of the disease. The histology of white dots in sympathetic ophthalmia, ${ }^{811}$ in Vogt-Koyanagi-Harada disease, ${ }^{12}$ and in sarcoidosis ${ }^{11}$ has been reported. The similarity between the white dots of sympathetic ophthalmia and sarcoid uveitis has been reported previously. ${ }^{13} 14$ Characteristically, the lesion appears as a small granuloma (microgranuloma) composed of lymphocytes and macrophages. In some cases the 'white dot' lesion lies at the choroidoretinal interface while in others it occurs as a discrete granuloma within the choroid (Fig 1g, h). Lesions which occur at the choroidoretinal interface at the level of the RPE may be situated between Bruch's membrane and the RPE or between the RPE and the photoreceptors in the subretinal space.

Immunohistologically, white dot lesions have a highly consistent composition irrespective of whether they are sited at the choroidoretinal interface or deeper in the choroid. ${ }^{913}{ }^{15-17} \mathrm{CD} 4+\mathrm{T}$ cells are the major lymphocyte subset in granulomas, with less than $10 \% \mathrm{CD} 8+\mathrm{T}$ cells in the lesions, and they form a rim in the periphery of the granuloma, with macrophages and other MHC class II positive cells forming the centre or core. B cells are not a major component of these lesions (Fig li, j, k).

The consistent cellular composition despite the variable location within the choroid/retina suggest strongly that the pathogenic mechanisms which generate these lesions are the same. In addition, the elegant clinicopathological study by Sharp et al ${ }^{11}$ correlating the characteristic fluorescein angiographic appearances with the histology of the white dot provides clear evidence for the nature of these lesions. We suggest that the early hypofluorescence of the white dot is due to dye exclusion within the cellular core of the lesion while the later hyperfluorescence is due to exudation and trapping of plasma/serum components at the site of focal inflammation. These findings have been reinforced by the recent observations of other groups using fluorescein angiography in $\mathrm{AMPPE}{ }^{18}$ and in MEWDS. ${ }^{19}$

\section{Pathogenesis of white dots}

Granuloma formation is the hallmark of the delayed type hypersensitivity (DTH) response initiated by the presentation of antigen, either foreign or self antigen, by antigen presenting cells (APCs) to Th1 type CD4+ T cells. Typical APCs include macrophages, $B$ cells, and dendritic cells of which dendritic cells are the most potent. ${ }^{20} \mathrm{~B}$ cells do not appear to be involved in granuloma formation since they preferentially present antigen to Th2 type CD4+ T cells. In contrast, all granulomas, including the 'white dots' of the retina/choroid, are populated by macrophages and dendritic cells (Fig $1 \mathrm{e}-\mathrm{h}$ ) and in certain circumstances such as in chronic granuloma formation of sarcoidosis, the granuloma has been likened to a small antigen presentation 'factory'.21

Why do granulomas form? APCs, particularly dendritic cells, which have been activated by an appropriate antigen, avidly bind $T$ cells and induce $T$ cell blast responses such as $T$ cell proliferation and release of pro-inflammatory cytokines and chemokines. This attracts further APCs to the evolving cell cluster and eventually leads to a granuloma. Specific cytokines are involved, such as interleukin 1 (IL-1), interferon $\gamma$ (IFN $\gamma$ ), tumour necrosis factor $\alpha$ (TNF $\alpha$ ), granulocyte-macrophage colony stimulation factor (GM-CSF), monocyte chemotactic protein-1 
(MCP-1), and the chemokine RANTES. ${ }^{22-26}$ RANTES is particularly important in this process since it appears to be specific for inducing chemotaxis of memory $\mathrm{T}$ cells and to be required for the development of granulomatous DTH responses. ${ }^{27}$

In the normal choroid, dendritic cells and macrophages are distributed widely as rich networks of potential APCs. ${ }^{28}$ Dendritic cells are derived from the bone marrow and traffic through the tissues in the role of the 'sentinel' cells - that is, their function is to detect foreign and altered self antigens and organise removal of these antigens from the tissues. ${ }^{20}$ Choroidal dendritic cells are large veil-like cells which occur at several levels within the choroid particularly in association with vessels but also in the immediate vicinity of the RPE where they are thought to act as antigen 'traps'. They are, therefore, ideal candidates to act as initiators of the 'white dot' granulomas at the choroidoretinal interface or deeper in the choroid. They might be activated singly or simultaneously, in large groups, depending on the nature and amount of antigen which they had to deal with, and they would disappear once they had destroyed the offending antigen. Alternatively, they would persist if the antigen was not readily removed. In the latter case there would be recruitment of further APCs and T cells with extension of the granuloma or white dot. Since choroidal dendritic cells are distributed uniformly throughout the choroid, it can be envisaged that for each actively functioning dendritic cell there would develop a corresponding white dot (Fig 11).

We suggest, therefore, that inflammatory diseases affecting the RPE/choroid and caused by foreign or self antigens, present clinically as focal or multifocal 'white dot' lesions because of the focal distribution of the antigen presenting dendritic cells which are required to initiate the development of the microgranuloma. Clinically, this might explain why white dots appear white when illuminated with white light since they represent accumulations of white cells, from which blood vessels and therefore red cells have been excluded. Each white dot granuloma progresses through several stages until either it vanishes without trace with restoration of the normal choroidoretinal architecture or it leaves a healed 'punched out' avascular scar. Each of these outcomes depends on how readily the inflammatory reaction is downregulated.

It might be asked, since dendritic cells and macrophages appear to be so numerous in the uveal tract, why 'white dots' and similar posterior ocular inflammatory disorders do not occur more frequently. It is possible that such events occur but they go undetected because they are so short lived that they do not produce symptoms. Indeed, many of the white dots are undetectable even by ophthalmoscopy and only become apparent in the late phases of a fluorescein angiogram (Fig 2).

However, under normal circumstances chorioretinal inflammation does not readily occur. This is probably due to the effectiveness of immunoregulatory mechanisms which operate within the eye to ensure that sight damaging inflammatory responses are kept to a minimum. ${ }^{29}$ In the posterior segment, Muller cells and RPE cells release a range of immunosuppressive factors such as $\mathrm{PGE}_{2}$ and nitric oxide when stimulated by cytokines released from $T$ cells and monocytes. ${ }^{3031}$ However, if stimulated by certain combinations of cytokines such as IL- 1 and IFN $\gamma$, RPE cells may act in a pro-inflammatory manner by releasing factors such as IL-8, MCP-1, and RANTES, thus encouraging the development of granulomas at the site of the $\mathrm{RPE}^{32}$ (Kuppner et al submitted). In contrast, RPE cells, when activated by IL-1 in synergy with immunosuppressive cytokines such as TGF $\beta$, release cytokines such as IL-6 which is thought to be important in downregulating the inflammatory response and limiting tissue damage. ${ }^{32}$ It is, therefore, clear that the precise cytokine profile within the inflammatory microenvironment of the retina/choroid determines whether the inflammation subsides or progresses and whether the white dot shows an evanescent characteristic or not.

\section{Conclusion}

From these clinical, pathological, and experimental observations the possibility that fundal white dots are the clinical expression of the formation of microgranulomata is suggested. 689 Their size, configuration, and pattern (whether evanescent or persistent) depend on the outcome of interactions between triggering factors involved in presentation of antigen (foreign or self) and the host immune system. A well regulated response mediated by appropriate cytokine release locally would be reflected by the formation of small, discrete, evanescent lesions while a poorly controlled response mediated by continued release of proinflammatory cytokines would require the mobilisation of additional cellular elements and the formation of larger poorly defined white dots which persisted in a state of chronic activity with permanent tissue destruction (scarring and fibrosis).

These observations have clinical implications. At one end of the spectrum the mild evanescent lesion (as in MEWDS) does not require treatment since the normal immunoregulatory mechanisms within the eye are adequate to contain the inflammation. However, at the other end of the spectrum, severe unrelenting inflammation, as in birdshot retinochoroidopathy and sympathetic ophthalmia, may cause extensive retinal damage and loss of vision. In these cases appropriate immunosuppressive treatment is required. ${ }^{33}$

This paper in part summarises work supported by grants from the Guide Dogs for the Blind (UK), the Royal National Institute for the Blind (UK) and the Welcome Trust to JVF.

Department of Ophthalmology,

DAVID BEN EZRA

Hadassah Medical Organisation,

Jerusalem, Israel

Department of Ophthalmology,

University of Aberdeen,

Scotland

1 Jampol LM, Sieving PA, Pugh D, Fishman GA, Gilbert H. Multiple evanescent white dot syndrome. Arch Ophthalmol 1984; 102: 671-4.

2 Aaberg TM, Campo RV, Joffe L. Recurrences and bilaterality in the multiple evanescent white dot syndrome. Am f Ophthalmol 1985; 100: 29-37.

Wyhinny GJ, Jackson JL, Jampol LM. Subretinal neovascularisation following multiple evanescent white dot syndrome. Arch Ophthalmol 1990; 108: 1384-8.

4 Sieving PA, Fishman GA, Jampol LM, Pugh D. Multiple evanescent white dot syndrome. II Electrophysiology of the photoreceptors during retinal pigment epithelial disease. Arch Ophthalmol 1984; 102: 675-9.

5 van Meel GJ, Kennen JEE, van Norren D, vande Kraats J. Scanning lase densitometry in multiple evanescent white dot syndrome. Retina 1993; 13: 29-35.

6 BenEzra D. The white dot - A spectrum of the same event in uveitis. XII Congress of the Asia Pacific Academy of Ophthalmology 1991 (abstract).

7 Gass JDM. Acute posterior multifocal placoid pigment epitheliopathy. Arch Ophthalmol 1968;80: 177-85.

8 Forrester JV, Liversidge JM, Dua HS, Towler HM, McMenamin PG Comparison of clinical and experimental uveitis. Curr Eye Res 1990; 9 (suppl): 75-84

9 Forrester JV. Duke Elder lecture: new concepts on the role of autoimmunity in the pathogenesis of uveitis. Eye 1992; 6: 433

10 Forrester JV. Uveitis. Br $\mathcal{F}$ Ophthalmol 1990; 74: 620-2.

11 Sharp DC, Bell RA, Patterson E, Pinkerton RMH. Sympathetic ophthalmia: histopathologic and fluorescein angiographic correlation. Arch Ophthalmol 1984; 102: 232-5.

12 Inomata $\mathrm{H}$. Necrotic changes of choroidal melanocytes in sympathetic ophthalmia. Arch Ophthalmol 1988; 106: 239-42.

13 Chan C-C, BenEzra D, Rodrigues MM, Palestine AG, Hsu SM Nussenblatt RB. Immunohistology and electronmicroscopy of choroida infiltrates and Ophthalmology 1985; 92: 580-5.

14 Chan C-C, Ben Ezra D, Hsu S-M, Palestine AG, Nussenblatt RB. Granulomas in sympathetic ophthalmia and sarcoidosis. Arch Ophthalmol 1985; 103: 198-202. 
15 Chan C-C, Palestine MR, Kuwabara T, Nussenblatt RB. Immunohistopathology of ocular sarcoidosis. Arch Ophthalmol 1987; 105: 1398-402.

16 Inomata H, Sakamoto T. Immunohistochemical studies of Vogt-KoyanagiHarada disease with sunset sky fundus. Curr Eye Res 1990; 95: 35-40.

17 Jakobiec F, Marboe C, Knowles D, Iwamoto T, Harrison W, Chang S, Coleman D. Human sympathetic ophthalmia. Ophthalmology 1983; 90: Coleman D. Human sympathetic ophthalmia. Ophthalmology 1983; 90 8 76-95. tifocal placoid pigment epitheliopathy. An indocyanine green angiographic study. Retina 1993; 13: 317-22.

19 Ie D, Glaser BM, Murphy RP, Gordon LW, Sjaarda RN, Thompson JT. Indocyanine green angiography in multiple evanescent white-dot syndrome. Am $f$ Ophthalmol 1994; 117: 7-12.

20 Steinman RM. The dendritic cell system and its role in immunogenicity. Annu Rev Immunol 1991; 9: 271-96.

21 Forrester JV. Sarcoidosis and inflammatory eye disease [Editorial]. $\mathrm{Br} \mathcal{F}$ Ophthalmol 1992; 76: 193.

22 Baggiolini M, Dewald B, Moser B. Interleukin 8 and related chemotactic cytokines-CXC and CC chemokines. Adv Immunol 1994; 55: 97-178. 23 di Giovanne F, Duff GW. Interleukin 1: the first interleukin. Immunol Today 1990; 11: 13 .

24 Dinarello CA. Interleukin-1 and interleukin-1 antagonism. Blood 1991; 77: $1627-52$.

25 Lonnemann G, Endres S, van der Meer JWM, Cannon JG, Koch KM, Dinarello CA. Differences in the synthesis and kinetics of release of interleukin 1a, interleukin $1 \mathrm{~b}$ and tumour necrosis factor from human mononuclear cells. Eur f Immunol 1989; 19: 1531-6.
26 Ruef C, Coleman DL. Granulocyte-macrophage colony stimulating factor: pleiotropic cytokine with potential clinical usefulness. Rev Infect Dis 1990 12: $41-62$.

27 Forrester JV, Lumsden L, Liversidge J, Kuppner M, Mesri M Immunoregulation at the choroido-retinal interface. Prog Retinal Eye Res 1995 (in press).

28 Forrester JV, McMenamin PG, Holthouse I, Lumsden L, Liversidge J. Localisation and characterisation of major histocompatibility complex Class II-positive cells in the posterior segment of the eye: implications for the induction of autoimmune uveoretinitis. Invest Ophthalmol Vis Sci 1994; 35: 64-77.

29 Streilein JW, Willbanks GA, Cousins SW. Immunoregulatory mechanisms of the eye. $₹$ Neuroimmunol 1992; 39: 185-200.

30 Liversidge J, Grabowski P, Ralston S, Benjamin N, Forrester JV. Rat retinal pigment epithelial cells express an inducible form of nitric oxide synthase and produce nitric oxide in response to inflammatory cytokines and activated T cells. Immunology 1994; 83: 404-9.

31 Liversidge JL, McKay D, Mullen G, Forrester JV. Retinal pigment epithelial cells modulate lymphocyte function at the blood-retina barrier by autocrine PGE2 and membrane bound mechanisms. Cell Immunol 1993; 149: 315-30.

32 Kuppner MC, McKillop-Smith S, Forrester JV. Transforming growth factor $b$ and IL-1 act in synergy to enhance IL 6 and IL 8 mRNA levels and IL6 production by human retinal pigment epithelial cells. Immunology 1995; 84: 265-71.

33 BenEzra D, Nussenblatt RB, Timonen P. The optimal use of Sandimmune in endogenous uveitis. [monograph] Berlin-Heidelberg: Springer-Verlag, 1988 . 